\title{
MEAN NUMBER OF REAL ZEROS OF A RANDOM TRIGONOMETRIC POLYNOMIAL
}

\author{
J. ERNEST WILKINS, JR.
}

\author{
(Communicated by William D. Sudderth)
}

\begin{abstract}
If $a_{1}, a_{2}, \ldots, a_{n}$ are independent, normally distributed random variables with mean 0 and variance 1 , and if $\nu_{n}$ is the mean value of the number of zeros on the interval $(0,2 \pi)$ of the trigonometric polynomial $a_{1} \cos x+$ $a_{2} \cos 2 x+\cdots+a_{n} \cos n x$, then

$\nu_{n}=3^{-1 / 2}\left\{(2 n+1)+D_{1}+(2 n+1)^{-1} D_{2}+(2 n+1)^{-2} D_{3}\right\}+O\left\{(2 n+1)^{-3}\right\}$, in which $D_{1}=0.232423 \cdots, D_{2}=-0.25973 \cdots$, and $D_{3}=0.2172 \cdots$.
\end{abstract}

\section{INTRODUCTION}

Suppose that $a_{1}, a_{2}, \ldots, a_{n}$ are independent, normally distributed random variables with mean 0 and variance 1 , and that $\nu_{n}$ is the mean value of the number of zeros on the interval $(0,2 \pi)$ of the trigonometric polynomial $a_{1} \cos x+$ $a_{2} \cos 2 x+\cdots+a_{n} \cos n x$. Das [5] has shown that $\nu_{n}=\left(2 n / 3^{1 / 2}\right)+O\left(n^{1 / 2}\right)$ for large $n$. Christensen and Sambandham [3] (cited by Bharucha-Reid and Sambandham [2, p. 114]) have shown that $\left|\nu_{n}-\left(2 n / 3^{1 / 2}\right)\right| \leq 10 n^{1 / 2}$ when $n \geq 25$. In this paper we will show that the error term $O\left(n^{1 / 2}\right)$ in the Das result is actually $O(1)$. More precisely, we will prove the following theorem:

Theorem. For large $n$, it is true that

$$
\nu_{n}=3^{-1 / 2}(2 n+1) \sum_{r=0}^{3}(2 n+1)^{-r} D_{r}+O\left\{(2 n+1)^{-3}\right\},
$$

in which $D_{0}=1$ and $D_{1}, D_{2}$, and $D_{3}$ are explicitly defined constants (see Equations (3.57), (3.58), and (3.59)) whose numerical values are approximately $0.232423,-0.25973$, and 0.2172 , respectively.

After a statement of the basic formulas on which our analysis rests, we devote $\S 2$ to the derivation of a convergent series representation of $\nu_{n}$ (cf. Lemma 7). Asymptotic representations for the first few coefficients in that series are derived in $\S 3$ and are used to establish the Theorem. We have calculated 7S values of $\nu_{n}$

Received by the editors November 20, 1989 and, in revised form, January 26, 1990.

1980 Mathematics Subject Classification (1985 Revision). Primary 60G99. 
when $n=2(1) 80$. In $\S 4$ we use these values to assess the accuracy of (1.1). In particular, the approximation to $\nu_{n}$ obtained from $(1.1)$ if the $O\left\{(2 n+1)^{-3}\right\}$ term is neglected produces $7 \mathrm{~S}$ values that differ from the calculated values by at most $10^{-5}$ when $n \geq 12$ and by only about $(2 / 15) \%$ when $n$ is as small as 2 .

\section{Preliminary analysis}

It is known $[2$, p. $105 ; 4$, p. 285] that, when $n \geq 2$,

$$
\nu_{n}=\pi^{-1} \int_{0}^{2 \pi} F_{n}(x) d x
$$

where

$$
\begin{gathered}
F_{n}=A_{n}^{-1}\left(A_{n} C_{n}-B_{n}^{2}\right)^{1 / 2}, \\
A_{n}=\sum_{j=1}^{n} \cos ^{2} j x, B_{n}=\sum_{j=1}^{n} j \sin j x \cos j x, C_{n}=\sum_{j=1}^{n} j^{2} \sin ^{2} j x .
\end{gathered}
$$

It is then obvious that

$$
\nu_{n}=4 \pi^{-1} \int_{0}^{\pi / 2} F_{n}(x) d x .
$$

We will need the explicit representations of $A_{n}, B_{n}$, and $C_{n}$ stated in the following lemma.

Lemma 1. It is true that

$$
\begin{gathered}
4 A_{n}=(2 n+1) g_{0}+g_{1}, \\
8 B_{n}=(2 n+1)^{2} h_{0}+(2 n+1) h_{1}+h_{2}, \text { and } \\
48 C_{n}=(2 n+1)^{3} k_{0}+(2 n+1)^{2} k_{1}+(2 n+1) k_{2}+k_{3},
\end{gathered}
$$

if the quantities $g_{0}, g_{1}, h_{0}, h_{1}, h_{2}, k_{0}, k_{1}, k_{2}$, and $k_{3}$ are defined so that

$$
\begin{gathered}
g_{0}=1+z^{-1} \sin z=4 \int_{0}^{1 / 2} \cos ^{2} z y d y, \\
g_{1}=-2+f(x) \sin z, \quad f(x)=c s c x-x^{-1}, \\
h_{0}=-d g_{0} / d z=4 \int_{0}^{1 / 2} y \sin 2 z y d y=-z^{-1} \cos z+z^{-2} \sin z, \\
h_{1}=-f(x) \cos z, \quad h_{2}=-f^{\prime}(x) \sin z, \\
k_{0}=1-3 d h_{0} / d z=48 \int_{0}^{1 / 2} y^{2} \sin ^{2} z y d y \\
=1-3 z^{-1} \sin z-6 z^{-2} \cos z+6 z^{-3} \sin z,
\end{gathered}
$$


(2.13) $k_{1}=-3 f(x) \sin z, \quad k_{2}=6 f^{\prime}(x) \cos z-1, \quad k_{3}=3 f^{\prime \prime}(x) \sin z$, and

$$
z=(2 n+1) x .
$$

It is a consequence of a known result $[6$, p. 82; 8, p. 177] (or of a simple mathematical induction) that

$$
4 A_{n}=2 n-1+\csc x \sin z .
$$

The validity of (2.5), together with (2.8) and (2.9), is now obvious. The validity of (2.6) together with (2.10) and (2.11), and of (2.7) together with (2.12) and (2.13), follows at once from (2.5) and the observations that

$$
\begin{gathered}
2 B_{n}=-d A_{n} / d x, \\
48 C_{n}=24 \sum_{j=1}^{n} j^{2}-24 d B_{n} / d x=(2 n+1)^{3}-(2 n+1)-24 d B_{n} / d x .
\end{gathered}
$$

For future reference we record in the following lemma certain properties of the function $f(x)[1$, pp. 75, 804, 805].

Lemma 2. If $\beta_{2 m}$ is the Bernoulli number of order $2 m$, then

$$
\begin{aligned}
f(x) & =\sum_{m=1}^{\infty}(-1)^{m-1}\left(2^{2 m}-2\right) \beta_{2 m} x^{2 m-1} /(2 m) ! \\
& =(x / 6)+\left(7 x^{3} / 360\right)+\left(31 x^{5} / 15120\right)+\cdots .
\end{aligned}
$$

The power series in (2.17) converges when $|x|<\pi$, and its coefficients are positive.

We calculate the first factor $A_{n}^{-1}$ in (2.2) in the following lemma:

Lemma 3. It is true that

$$
\left(4 A_{n}\right)^{-1}=\left\{(2 n+1) g_{0}\right\}^{-1} \sum_{r=0}^{\infty}(2 n+1)^{-r}\left(-g_{1} / g_{0}\right)^{r} .
$$

The series in (2.18) converges absolutely and uniformly when $0 \leq x \leq \pi / 2$, $n \geq 2$.

The absolute minimum value of $z^{-1} \sin z$ (when $0<z<\infty$ ) is attained when [7, p. 4] $z=4.4934095 \cdots$, and is greater than -0.2172337 . The maximum value of $f(x)$ (when $0 \leq x \leq \pi / 2$ ) is $f(\pi / 2)$, or $1-2 \pi^{-1}$ (because the coefficients in the power series (2.17) are positive). We infer from (2.8) and (2.9) that $g_{0}>0.7827663$ and $0<-g_{1} \leq 3-2 \pi^{-1}<2.363381$. Lemma 3 is now a consequence of the observation that $0<(2 n+1)^{-1}\left(-g_{1} / g_{0}\right)<0.604<1$ when $n \geq 2$.

A straightforward calculation, based on Equations (2.5) through (2.13), shows 
the validity of the following lemma:

Lemma 4. It is true that

(2.19) $192\left(A_{n} C_{n}-B_{n}^{2}\right)=(2 n+1)^{4} f_{0}+(2 n+1)^{3} f_{1}+(2 n+1)^{2} f_{2}+(2 n+1) f_{3}+f_{4}$, if the quantities $f_{0}, f_{1}, f_{2}, f_{3}$, and $f_{4}$ are defined so that

(2.20) $f_{0}=g_{0} k_{0}-3 h_{0}^{2}$

$$
=1-2 z^{-1} \sin z-3 z^{-2}(1+2 \cos z)+6 z^{-3} \sin z+3 z^{-4} \sin ^{2} z,
$$

(2.21) $f_{1}=g_{0} k_{1}+g_{1} k_{0}-6 h_{0} h_{1}$

$$
=-2\left\{k_{0}+\left(\sin z+3 z^{-1}-3 z^{-3} \sin ^{2} z\right) f\right\},
$$

$$
\text { (2.22) } \begin{aligned}
f_{2} & =g_{0} k_{2}+g_{1} k_{1}-3 h_{1}^{2}-6 h_{0} h_{2} \\
& =-1+6 f \sin z+6 f^{\prime} \cos z-3 f^{2}-z^{-1} \sin z+6 f^{\prime} z^{-2} \sin ^{2} z, \\
\text { (2.23) } f_{3} & =g_{0} k_{3}+g_{1} k_{2}-6 h_{1} h_{2} \\
& =2-f \sin z-12 f^{\prime} \cos z+3 f^{\prime \prime} \sin z+3 f^{\prime \prime} z^{-1} \sin ^{2} z,
\end{aligned}
$$

and

$$
f_{4}=g_{1} k_{3}-3 h_{2}^{2}=3\left(f f^{\prime \prime}-f^{\prime 2}\right) \sin ^{2} z-6 f^{\prime \prime} \sin z .
$$

We calculate the second factor $\left(A_{n} C_{n}-B_{n}^{2}\right)^{1 / 2}$ in (2.2) in the following lemma:

Lemma 5. There exists an integer $n_{0}$ such that $n_{0} \geq 2$

$$
(192)^{1 / 2}\left(A_{n} C_{n}-B_{n}^{2}\right)^{1 / 2}=(2 n+1)^{2} f_{0}^{1 / 2} \sum_{r=0}^{\infty}(2 n+1)^{-r} c_{r}
$$

when $n \geq n_{0}$, in which $b_{r}=f_{r} / f_{0}(r=0,1, \ldots, 4)$, and

$$
\begin{aligned}
& c_{0}=1, \\
& c_{1}=b_{1} / 2, \\
& c_{2}=\left(b_{2} / 2\right)-\left(b_{1}^{2} / 8\right), \\
& c_{3}=\left(b_{3} / 2\right)-\left(b_{1} b_{2} / 4\right)+\left(b_{1}^{3} / 16\right), \cdots .
\end{aligned}
$$

The series in (2.25) converges absolutely and uniformly when $0 \leq x \leq \pi / 2$, $n \geq n_{0}$.

It is a consequence of the representations of $g_{0}, h_{0}$, and $k_{0}$ as integrals in (2.8), (2.10), (2.12), and the Schwarz inequality that

$$
f_{0} / 192=\int_{0}^{1 / 2} \cos ^{2} z y d y \int_{0}^{1 / 2} y^{2} \sin ^{2} z y d y-\left\{\int_{0}^{1 / 2} y \sin z y \cos z y d y\right\}^{2}>0
$$

when $z>0$. Moreover, $f_{0}=\left(4 z^{2} / 15\right)+O\left(z^{4}\right)$ for small $z$ and $f_{0}=1+O\left(z^{-1}\right)$ for large $z$. It follows from Lemma 2 that $x^{-1} f(x), x^{-2}\left\{6 f^{\prime}(x)-1\right\}$, and $x^{-1} f^{\prime \prime}(x)$ are bounded when $0 \leq x \leq \pi / 2$. Because $x=z /(2 n+1) \leq z / 5$ 
when $n \geq 2$, an inspection of Equations (2.21) through (2.25) now shows that $f_{r}=O\left(z^{2}\right)$ when $z$ is small, and that $f_{r}=O(1)$ for all $z$. This remark suffices to justify the following identity:

$$
192\left(A_{n} C_{n}-B_{n}^{2}\right)=(2 n+1)^{4} f_{0} \sum_{r=0}^{4}(2 n+1)^{-r} b_{r},
$$

in which $b_{r}=f_{r} / f_{0}$. We may also conclude that $b_{r}=O(1)$, uniformly in $x$ and $n$ when $0 \leq x \leq \pi / 2, n \geq 2$.

Let $\rho$ be a fixed constant such that $0<\rho<1$. Then there surely exists an integer $n_{0}$ such that $n_{0} \geq 2$ and

$$
\sum_{r=1}^{4}(2 n+1)^{-r}\left|b_{r}\right|<\rho
$$

when $n \geq n_{0}$ and $0 \leq x \leq \pi / 2$. Lemma 5 is now a consequence of (2.28) and (2.29).

If we use (2.2) and Lemmas 3 and 5, we obtain the following lemma:

Lemma 6. It is true that, when $n \geq n_{0}$,

$$
F_{n}(x)=(2 n+1)\left(f_{0} / 12\right)^{1 / 2} g_{0}^{-1} \sum_{r=0}^{\infty}(2 n+1)^{-r} u_{r},
$$

in which

$$
u_{r}=\sum_{s=0}^{r}\left(-g_{1} / g_{0}\right)^{s} c_{r-s}
$$

The series in (2.30) converges absolutely and uniformly when $0 \leq x \leq \pi / 2$, $n \geq n_{0}$.

The following lemma is now a consequence of (2.4) and Lemma 6:

Lemma 7. It is true that, when $n \geq n_{0}$,

$$
\nu_{n}=3^{-1 / 2}(2 n+1) \sum_{r=0}^{\infty}(2 n+1)^{-r} v_{r}
$$

in which

$$
\begin{gathered}
v_{r}=2 \pi^{-1} \int_{0}^{\pi / 2} G(z) u_{r} d x \text { and } \\
G(z)=f_{0}^{1 / 2} g_{0}^{-1} .
\end{gathered}
$$

\section{Proof of the theorem}

In the next four lemmas we will exhibit constants $S_{r m}(0 \leq r+m \leq 3)$ and $S_{r}(r=0,1,2,3)$ such that

$$
v_{r}=\sum_{m=0}^{3-r}(2 n+1)^{-m} S_{r m}+(-1)^{n}(2 n+1)^{r-3} S_{r}+O\left\{(2 n+1)^{r-4}\right\}
$$


when $r=0,1,2,3$. In view of (2.32), this will be sufficient to prove (1.1) if

$$
D_{r}=\sum_{m=0}^{r} S_{r-m, m}(r=0,1,2,3), \sum_{r=0}^{3} S_{r}=0 .
$$

Lemma 8. Equation (3.1) is true when $r=0$ if

$$
\begin{aligned}
S_{00} & =1, \\
S_{01} & =2 \pi^{-1} \int_{0}^{\infty}\{G(z)-1\} d z, \\
S_{02} & =-3 \pi^{-2}, \\
S_{03} & =0, \\
S_{0} & =-8 \pi^{-3} .
\end{aligned}
$$

It follows from (2.33) and (2.31) when $r=0$, and (2.26) that

$$
\pi v_{0} / 2=(\pi / 2)+(2 n+1)^{-1} \int_{0}^{\lambda}\{G(z)-1\} d z,
$$

in which

$$
\lambda=(2 n+1) \pi / 2 .
$$

If $z$ is large, we deduce from the definitions (2.34), (2.8), and (2.20) of $G(z)$, $g_{0}$, and $f_{0}$, respectively, that

(3.6) $G(z)=1-2 z^{-1} \sin z-0.75 z^{-2}(1+4 \cos z+\cos 2 z)+z^{-3} T_{1}(z)+O\left(z^{-4}\right)$,

in which $T_{1}(z)$ is a trigonometric sine polynomial of degree 3 . It follows from (3.4), and (3.6) to ensure convergence, that

$$
\begin{aligned}
\pi v_{0} / 2= & (\pi / 2)+(2 n+1)^{-1} \int_{0}^{\infty}\{G(z)-1\} d z \\
& -(2 n+1)^{-1} \int_{\lambda}^{\infty}\{G(z)-1\} d z .
\end{aligned}
$$

Repeated integration by parts of the last term in (3.7) now shows, with the help of (3.6), that Lemma 8 is true.

Lemma 9. Equation (3.1) is true when $r=1$ if

$$
\begin{aligned}
& S_{10}=1, \\
& S_{11}=2 \pi^{-1} \int_{0}^{\infty}\left\{H_{1}(z)-1\right\} d z-3 \pi^{-1} \int_{0}^{\pi / 2} x^{-1} f(x) d x, \\
& S_{12}=3 \pi^{-2}-(3 \pi)^{-1} \int_{0}^{\infty} z\left\{H_{2}(z)-2 \sin z-3 z^{-1} \cos ^{2} z\right\} d z, \\
& S_{1}=-16 \pi^{-3}
\end{aligned}
$$

in which

$$
\begin{aligned}
H_{1}(z)=f_{0}^{-1 / 2} g_{0}^{-2}\left\{1-2 z^{-1} \sin z-1.5 z^{-2}(3\right. & +4 \cos z+\cos 2 z) \\
& \left.+3 z^{-3}(2 \sin z+\sin 2 z)\right\}
\end{aligned}
$$


and

(3.10)

$$
H_{2}(z)=f_{0}^{-1 / 2} g_{0}^{-2}\left\{2 \sin z+0.5 z^{-1}(5+\cos 2 z)-3 z^{-2} \sin 2 z+3 z^{-3} \sin ^{2} z\right\} \text {. }
$$

It follows from (2.31) when $r=1,(2.26)$, the definition of $b_{r}$ as $f_{r} / f_{0}$, and the definitions (2.8), (2.9), (2.20), and (2.21) of $g_{0}, g_{1}, f_{0}$, and $f_{1}$, respectively, that

$$
G(z) u_{1}=H_{1}(z)-H_{2}(z) f(x),
$$

if $H_{1}$ and $H_{2}$ are the functions defined in (3.9) and (3.10). We deduce from (3.9), (3.10) and the definitions (2.8) and (2.20) of $g_{0}$ and $f_{0}$ that, when $z$ is large,

$H_{1}(z)=1-3 z^{-1} \sin z-0.75 z^{-2}(1+4 \cos z+5 \cos 2 z)+z^{-3} T_{2}(z)+O\left(z^{-4}\right)$,

and

$$
H_{2}(z)=2 \sin z+3 z^{-1} \cos ^{2} z+6 z^{-2} \sin ^{3} z+O\left(z^{-3}\right),
$$

in which $T_{2}(z)$ is a trigonometric sine polynomial of degree 3 . With the help of an argument like that which led from (3.4) through (3.6) to the proof of Lemma 8, we see that

$$
\begin{aligned}
\int_{0}^{\pi / 2} H_{1}(z) d x= & (\pi / 2)+(2 n+1)^{-1} \int_{0}^{\infty}\left\{H_{1}(z)-1\right\} d z+1.5 \pi^{-1}(2 n+1)^{-2} \\
& +O\left\{(2 n+1)^{-3}\right\} .
\end{aligned}
$$

Moreover,

$$
\int_{0}^{\pi / 2} H_{2}(z) f(x) d x=I_{1}+I_{2}+I_{3}+I_{4}+I_{5},
$$

in which

$$
\begin{aligned}
& I_{1}=\int_{0}^{\pi / 2}\left\{2 \sin z+1.5 z^{-1}(1+\cos 2 z)\right\} f(x) d x, \\
& I_{2}=\int_{0}^{\pi / 2}\left\{H_{2}(z)-2 \sin z-3 z^{-1} \cos ^{2} z\right\}(x / 6) d x,
\end{aligned}
$$

and

$$
\begin{aligned}
I_{5}= & \int_{0}^{\pi / 2}\left\{H_{2}(z)-2 \sin z-3 z^{-1} \cos ^{2} z-6 z^{-2} \sin ^{3} z\right\} \\
& \times\left\{f(x)-(x / 6)-\left(7 x^{3} / 360\right)\right\} d x .
\end{aligned}
$$


It is obvious that

$$
\int_{0}^{\pi / 2} f(x) z^{-1} d x=(2 n+1)^{-1} \int_{0}^{\pi / 2} x^{-1} f(x) d x,
$$

and repeated integration by parts shows that

$$
\int_{0}^{\pi / 2} f(x) \sin z d x=4(-1)^{n} \pi^{-2}(2 n+1)^{-2}+O\left\{(2 n+1)^{-4}\right\}
$$

and

$$
\int_{0}^{\pi / 2} f(x) z^{-1} \cos 2 z d x=O\left\{(2 n+1)^{-3}\right\} .
$$

It follows from (3.16), (3.21), (3.22), and (3.23) that

$$
\begin{aligned}
I_{1}= & 1.5(2 n+1)^{-1} \int_{0}^{\pi / 2} x^{-1} f(x) d x \\
& +8(-1)^{n} \pi^{-2}(2 n+1)^{-2}+O\left\{(2 n+1)^{-3}\right\} .
\end{aligned}
$$

We use (3.13), (3.5), (2.14), and (2.17) to deduce from (3.17), (3.18), and (3.20) that

$$
\begin{aligned}
& 6 I_{2}=(2 n+1)^{-2} \int_{0}^{\lambda} z\left\{H_{2}(z)-2 \sin z-3 z^{-1} \cos ^{2} z\right\} d z \\
& =(2 n+1)^{-2} \int_{0}^{\infty} z\left\{H_{2}(z)-2 \sin z-3 z^{-1} \cos ^{2} z\right\} d z+O\left\{(2 n+1)^{-4}\right\}, \\
& 360 I_{3}=(2 n+1)^{-4} \int_{0}^{\lambda} z^{3} O\left(z^{-3}\right) d z=O\left\{(2 n+1)^{-3}\right\},
\end{aligned}
$$

and

$$
I_{5}=\int_{0}^{\pi / 2} O\left(x^{5}\right) O\left(z^{-3}\right) d x=O\left\{(2 n+1)^{-3}\right\}
$$

If we define $g(x)$ so that $f(x)=(x / 6)+x^{2} g(x)$, then $g(x)$ is an odd, infinitely differentiable function. The reasoning that led to (3.22) now enables us to deduce from (3.19) that

$$
I_{4}=1.5(2 n+1)^{-2} \int_{0}^{\pi / 2} g(x)(3 \sin z-\sin 3 z) d x=O\left\{(2 n+1)^{-4}\right\} .
$$

We conclude from (3.15), (3.24), (3.25), (3.26), (3.27), and (3.28) that

$$
\begin{aligned}
& \int_{0}^{\pi / 2} H_{2}(z) f(x) d x=1.5(2 n+1)^{-1} \int_{0}^{\pi / 2} x^{-1} f(x) d x \\
& \quad+(2 n+1)^{-2}\left[8(--1)^{n} \pi^{-2}+6^{-1} \int_{0}^{\infty} z\left\{H_{2}(z)-2 \sin z-3 z^{-1} \cos ^{2} z\right\} d z\right] \\
& \quad+O\left\{(2 n+1)^{-3}\right\} .
\end{aligned}
$$

If we combine (3.14) and (3.29) and use (3.11) and (2.33), we see that Lemma 9 is true. 
Lemma 10. Equation (3.1) is true when $r=2$ if

$$
\begin{aligned}
& S_{20}=1-1.5 \pi^{-1} \int_{0}^{\pi / 2} f^{2}(x) d x, \\
& S_{21}=\pi^{-1} \int_{0}^{\infty}\left\{2 J_{1}(z)-2-\cos z\right\} d z-3 \pi^{-1} \int_{0}^{\pi / 2} x^{-1} f(x) d x, \\
& S_{2}=24 \pi^{-3}
\end{aligned}
$$

in which

$$
\begin{aligned}
2 J_{1}(z)= & f_{0}^{-1 / 2} g_{0}^{-1}\left(-1+\cos z-z^{-1} \sin z+z^{-2} \sin ^{2} z\right) \\
& -f_{0}^{-3 / 2} g_{0}^{-1} k_{0}^{2}-4 f_{0}^{-1 / 2} g_{0}^{-2} k_{0}+8 f_{0}^{1 / 2} g_{0}^{-3} .
\end{aligned}
$$

It follows from (2.31) when $r=2,(2.26)$, the definition of $b_{r}$ as $f_{r} / f_{0}$, and the definitions (2.8), (2.9), (2.20), (2.21), and (2.22) of $g_{0}, g_{1}, f_{0}, f_{1}$ and $f_{2}$, respectively, that

$$
G(z) u_{2}=J_{1}(z)+J_{2}(z) f(x)+J_{3}(z) f^{2}(x)+J_{4}(z)\left\{6 f^{\prime}(x)-1\right\},
$$

if $J_{1}(z)$ is the function defined in (3.31), and $J_{2}(z), J_{3}(z)$, and $J_{4}(z)$ are explicitly definable functions concerning which we need to know, by analogy with (3.6), (3.12), and (3.13), only that for all $z, J_{2}(z)$ is bounded and

$$
\begin{gathered}
J_{2}(z)=-3 \sin z-1.5 z^{-1}(1+5 \cos 2 z)+z^{-2} T_{3}(z)+O\left(z^{-3}\right), \\
J_{3}(z)=-0.75(1+\cos 2 z)-6 z^{-1} \sin ^{3} z+O\left(z^{-2}\right), \text { and } \\
2 J_{4}(z)=\cos z+O\left(z^{-2}\right)
\end{gathered}
$$

in which $T_{3}(z)$ is a trigonometric sine polynomial of degree 3 . We also need to note that (3.31) implies that $J_{1}(z)$ is bounded for all $z$ and, for large $z$, that

$$
2 J_{1}(z)=2+\cos z-13 z^{-1} \sin z+O\left(z^{-2}\right) .
$$

If for large $z$ we use (3.36), we find that

$$
\begin{aligned}
2 \int_{0}^{\pi / 2} J_{1}(z) d x= & +(2 n+1)^{-1}\left[(-1)^{n}+\int_{0}^{\infty}\left\{2 J_{1}(z)-2-\cos z\right\} d z\right] \\
& +O\left\{(2 n+1)^{-2}\right\}
\end{aligned}
$$


If we use (3.21), (3.22), and (3.23), it follows from (3.33) that (3.38)

$$
\begin{aligned}
\int_{0}^{\pi / 2} & J_{2}(z) f(x) d x=-\int_{0}^{\pi / 2}\left\{3 \sin z+1.5 z^{-1}(1+5 \cos 2 z)\right\} f(x) d x \\
& +\int_{0}^{\pi / 2}\left\{J_{2}(z)+3 \sin z+1.5 z^{-1}(1+5 \cos 2 z)\right\}\{f(x)-(x / 6)\} d x \\
& +\int_{0}^{\pi / 2}\left\{J_{2}(z)+3 \sin z+1.5 z^{-1}(1+5 \cos 2 z)\right\}(x / 6) d x \\
= & -1.5(2 n+1)^{-1} \int_{0}^{\pi / 2} x^{-1} f(x) d x+O\left\{(2 n+1)^{-2}\right\} \\
& +\int_{0}^{\pi / 2} O\left(z^{-2}\right) O\left(x^{3}\right) d x \\
& +6^{-1}(2 n+1)^{-2} \int_{0}^{\lambda}\left\{J_{2}(z)+3 \sin z+1.5 z^{-1}(1+5 \cos 2 z)\right\} z d z \\
= & -1.5(2 n+1)^{-1} \int_{0}^{\pi / 2} x^{-1} f(x) d x+O\left\{(2 n+1)^{-2}\right\} .
\end{aligned}
$$

With the help of some integration by parts, we also find from (3.34) and (3.35) that

$$
\begin{aligned}
4 \int_{0}^{\pi / 2} J_{3}(z) f^{2}(x) d x= & -\int_{0}^{\pi / 2}\left\{3(1+\cos 2 z)+6 z^{-1}(3 \sin z-\sin 3 z)\right\} f^{2}(x) d x \\
& +\int_{0}^{\pi / 2}\left\{4 J_{3}(z)+3(1+\cos 2 z)+24 z^{-1} \sin ^{3} z\right\} f^{2}(x) d x \\
= & -3 \int_{0}^{\pi / 2} f^{2}(x) d x+O\left\{(2 n+1)^{-2}\right\}+\int_{0}^{\pi / 2} O\left(z^{-2}\right) O\left(x^{2}\right) d x \\
= & -3 \int_{0}^{\pi / 2} f^{2}(x) d x+O\left\{(2 n+1)^{-2}\right\}
\end{aligned}
$$

$$
\begin{aligned}
2 \int_{0}^{\pi / 2} J_{4}(z)\left\{6 f^{\prime}(x)-1\right\} d x= & \int_{0}^{\pi / 2}\left\{6 f^{\prime}(x)-1\right\} \cos z d x \\
& +\int_{0}^{\pi / 2} O\left(z^{-2}\right) O\left(x^{2}\right) d x \\
= & \left(24 \pi^{-2}-1\right)(-1)^{n}(2 n+1)^{-1}+O\left\{(2 n+1)^{-2}\right\} .
\end{aligned}
$$

If we combine (3.37), (3.38), (3.39), and (3.40), and use (3.32) and (2.33), we find that Lemma 10 is true.

Lemma 11. Equation (3.1) is true when $r=3$ if

$$
S_{30}=2-1.5 \pi^{-1} \int_{0}^{\pi / 2} f^{2}(x) d x, \quad S_{3}=0 .
$$


It follows from (2.31) when $r=3,(2.26)$, the definition of $b_{r}$ as $f_{r} / f_{0}$, and the definitions (2.8), (2.9), (2.20), (2.21), (2.22), and (2.23) of $g_{0}, g_{1}$, $f_{0}, f_{1}, f_{2}$ and $f_{3}$, respectively, that

$$
G(z) u_{3}=K_{1}+K_{2} f+K_{3} f^{\prime \prime}+K_{4} f^{2}+\left(K_{5}+K_{6} f\right)\left(6 f^{\prime}-1\right)+K_{7} f^{3}
$$

in which $K_{1}, \ldots, K_{7}$ are explicitly definable functions concerning which we need to know only that $K_{1}$ is bounded and that

$$
\begin{gathered}
K_{1}=2-0.5 \cos z+z^{-1} T_{4}(z)+O\left(z^{-2}\right), \\
K_{2}=T_{5} \sin z+O\left(z^{-1}\right) \\
K_{3}=T_{6} \sin z+O\left(z^{-1}\right) \\
4 K_{4}=-3-15 \cos 2 z+O\left(z^{-1}\right), \\
2 K_{5}=\cos z+O\left(z^{-1}\right) \\
K_{6}=O\left(z^{-1}\right), \text { and } \\
K_{7}=-2 \sin ^{3} z+O\left(z^{-1}\right),
\end{gathered}
$$

in which $T_{4}(z)$ is a trigonometric sine polynomial of degree 2 and $T_{5}$ and $T_{6}$ are constants. It then follows by familiar arguments that

$$
\int_{0}^{\pi / 2} K_{1} d x=\pi+O\left\{(2 n+1)^{-1}\right\}
$$

$$
\int_{0}^{\pi / 2} K_{4} f^{2} d x=-0.75 \int_{0}^{\pi / 2} f^{2}(x) d x+O\left\{(2 n+1)^{-1}\right\},
$$

and the remaining terms in (3.42) contribute only $O\left\{(2 n+1)^{-1}\right\}$ to the value of $v_{3}$. This remark completes the proof of Lemma 11 .

We infer from Lemmas 8 through 11 that $S_{0}+S_{1}+S_{2}+S_{3}=0$, and then from (3.2) that (1.1) is valid if $D_{0}=1$, and

$$
\begin{gathered}
D_{1}=2 \pi^{-1} \int_{0}^{\infty}\{G(z)-1\} d z+1 \\
D_{2}=3 \pi^{-2}+2 \pi^{-1} \int_{0}^{\infty}\left\{H_{1}(z)-1\right\} d z-3 \pi^{-1} \int_{0}^{\pi / 2} x^{-1} f(x) d x+1 \\
-1.5 \pi^{-1} \int_{0}^{\pi / 2} f^{2}(x) d x
\end{gathered}
$$


and

$$
\begin{aligned}
D_{3}= & 3 \pi^{-2}-(3 \pi)^{-1} \int_{0}^{\infty} z\left\{H_{2}(z)-2 \sin z-3 z^{-1} \cos ^{2} z\right\} d z \\
& +\pi^{-1} \int_{0}^{\infty}\left\{2 J_{1}(z)-2-\cos z\right\} d z-3 \pi^{-1} \int_{0}^{\pi / 2} x^{-1} f(x) d x \\
& +2-1.5 \pi^{-1} \int_{0}^{\pi / 2} f^{2}(x) d x .
\end{aligned}
$$

The conditionally convergent infinite integrals in (3.52), (3.53), and (3.54) can be replaced by absolutely convergent infinite integrals with the help of the identity,

$$
1=2 \pi^{-1} \int_{0}^{\infty} z^{-1} \sin z d z
$$

and of the relations (3.6), (3.12), (3.13), and (3.36). In addition, it is clear that

$$
\int_{0}^{\pi / 2}\left\{f^{2}(x)+2 x^{-1} f(x)\right\} d x=\int_{0}^{\pi / 2}\left(\csc ^{2} x-x^{-2}\right) d x=2 \pi^{-1} \text {. }
$$

Therefore,

$$
\begin{gathered}
D_{1}=2 \pi^{-1} \int_{0}^{\infty}\left\{G(z)-1+2 z^{-1} \sin z\right\} d z-1, \\
D_{2}=2 \pi^{-1} \int_{0}^{\infty}\left\{H_{1}(z)-1+3 z^{-1} \sin z\right\} d z-2 \text {, and } \\
D_{3}=\pi^{-1} \int_{0}^{\infty}\left\{2 J_{1}(z)-2-\cos z+13 z^{-1} \sin z\right\} d z-5 \\
-(3 \pi)^{-1} \int_{0}^{\infty} z\left\{H(z)-2 \sin z-3 z^{-1} \cos ^{2} z-6 z^{-2} \sin ^{3} z\right\} d z .
\end{gathered}
$$

This completes the proof of the Theorem, except for the numerical values of $D_{1}, D_{2}$, and $D_{3}$.

\section{Numerical Results}

We calculated the four integrals in (3.57), (3.58), and (3.59) over the interval $(0,25 \pi)$ by Simpson's rule. The integrals over the interval $(25 \pi, \infty)$ were calculated using the asymptotic relations (3.6), (3.12), (3.13), and (3.36) for $G(z), H_{1}(z), H_{2}(z)$, and $J_{1}(z)$, respectively. In each case it was desirable to include additional terms in those relations. The results for the coefficients $D_{1}, D_{2}$, and $D_{3}$ are those recorded in the statement of the Theorem.

We have also numerically computed the integral in (2.4) when $n=2(1) 80$. The approximation $3^{-1 / 2}\left(2 n+1+D_{1}\right)$ is in error (in excess) by about $0.00100 \%$ when $n=80,0.00176 \%$ when $n=60,0.00391 \%$ when $n=40,0.0150 \%$ when $n=20,0.0558 \%$ when $n=10,0.192 \%$ when $n=5$, and only 
$0.698 \%$ when $n=2$. The more accurate approximation $3^{-1 / 2}\left\{2 n+1+D_{1}+\right.$ $\left.(2 n+1)^{-1} D_{2}\right\}$ produces $7 \mathrm{~S}$ results that differ from the calculated values by at most $10^{-5}$ (one unit in the last calculated decimal place) when $n \geq 47$, by at most $10^{-4}$ when $n \geq 18$, by at most $10^{-3}$ when $n \geq 6$, and by only 0.00905 , or about $0.302 \%$, when $n=2$. The most accurate approximation

$$
3^{-1 / 2}\left\{2 n+1+D_{1}+(2 n+1)^{-1} D_{2}+(2 n+1)^{-2} D_{3}\right\}
$$

produces $7 \mathrm{~S}$ results that differ from the calculated results by at most $10^{-5}$ when $n \geq 12$, by at most $10^{-4}$ when $n \geq 7$, by at most $10^{-3}$ when $n \geq 3$, and by 0.00403 , or about $0.134 \%$ when $n=2$.

Although it is not really necessary to know the value of the integer $n_{0}$ in Lemma 5 , we have found numerical bounds for $\left|b_{r}\right|$, when $0 \leq x \leq \pi / 2$, $n \geq 2$, which imply that the inequality (2.29) is true if $\rho=0.93$. Therefore, Lemma 5 is true when $n_{0}=2$.

\section{REFERENCES}

1. M. Abramowitz and I. A. Stegun, eds., Handbook of mathematical functions, with formulas, graphs and mathematical tables, Wiley, New York, 1972.

2. A. T. Bharucha-Reid and M. Sambandham, Random polynomials, Academic Press, Orlando, 1986.

3. M. J. Christensen and M. Sambandham, Improved estimates for real roots of random trigonometric polynomials, unpublished manuscript.

4. H. Cramer and M. R. Leadbetter, Stationary and related stochastic processes, Wiley, New York, 1967.

5. M. Das, The average number of real zeros of a random trigonometric polynomial, Proc. Cambridge Philos. Soc. 64 (1968), 721-729.

6. H. T. Davis, The summation of series, Principia Press of Trinity University, San Antonio, 1962.

7. F. W. J. Olver, ed., Bessel functions. Part III. Zeros and associated values, Royal Society Mathematical Tables, vol. 7, Cambridge University Press, Cambridge, 1960.

8. E. T. Whittaker and G. N. Watson, A course of modern analysis (American ed.), MacMillan, New York, 1943.

4800 S. LaKe Shore Drive, Chicago, Illinois 60615 